\title{
Dimensional Regularization of Renyi's Statistical Mechanics
}

\author{
A. Plastino ${ }^{1,4,5}$, M. C. Rocca ${ }^{1,2,4}$, G. L. Ferri ${ }^{3}$, \\ 1 Departamento de Física, Universidad Nacional de La Plata, \\ 2 Departamento de Matemática, Universidad Nacional de La Plata, \\ ${ }^{3}$ Fac. de C. Exactas-National University La Pampa, \\ Peru y Uruguay, Santa Rosa, La Pampa, Argentina \\ ${ }^{4}$ Consejo Nacional de Investigaciones Científicas y Tecnológicas \\ (IFLP-CCT-CONICET)-C. C. 727, 1900 La Plata - Argentina \\ ${ }^{5}$ SThAR - EPFL, Lausanne, Switzerland
}

October 10, 2018

\begin{abstract}
We show that typical Renyi's statistical mechanics' quantifiers exhibit poles. We are referring to the partition function $\mathcal{Z}$ and the mean energy $\langle\mathcal{U}\rangle$. Renyi's entropy is characterized by a real parameter $\alpha$. The poles emerge in a numerable set of rational numbers belonging to the $\alpha$-line. Physical effects of these poles are studied by appeal to dimensional regularization, as usual. Interesting effects are found, as for instance, gravitational ones.
\end{abstract}

PACS: 05.20.-y, 02.10.-v 


\section{Introduction}

Renyi's information measure $S_{R}$ is a generalization of both Hartley's and Shannon's entropic quantifiers of our ignorance regarding a system's structural characteristics. $S_{\mathrm{R}}$ is regarded as a quite important measure in several science's areas. We may cite, for example, ecology, quantum information, Heisenberg's XY spin chain model, theoretical computing, conformal field

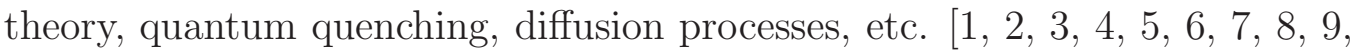
10, 11, 12.

The Renyi entropy is also relevant in statistics as signaling diversity. $S_{R}$ is defined as [1]:

$$
S_{R}=\frac{1}{1-\alpha} \ln \left(\int_{M} P^{\alpha} d \mu\right) .
$$

We will investigate here poles that emerge in computing the most important Renyi's statistical quantities for the harmonic oscillator ( $\mathrm{HO})$. We wish to ascertain the physical significance of these poles.

To such an end we appeal to the dimensional regularization methodology of Bollini and Giambiagi [13, [14, 15, 16], plus its generalization, developed in [17. Dimensional Regularization is one of the most important advances in theoretical physics and is used in several disciplines of it [18]-[71].

Why the HO? We do not mean to unravel HO's peculiarities here. This is a very well known system already, of course, described by $\alpha=1$ (when $S_{R}$ becomes Shannon's entropy). We use the HO because of its simplicity, so that closed-form formulas become available. This enormously facilitates our pole-research and we thus obtain indications as of how to proceed in more complex situations. Our present work is an unavoidable preliminary step to be taken before tackling such situations.

We will separately treat the one, two, and three dimensions cases, as the poles are different for each dimension. We start below with some general considerations and will heavily rely on reference [72], which should be recommended as a useful prerequisite. 


\section{Theoretical considerations for the $\alpha$-region outside the poles}

\section{$2.1 \alpha>1$}

We showed in [72] that the classical Renyi-HO partition function is, for $\alpha>1$,

$$
\mathcal{Z}=\frac{\pi^{v}}{\Gamma(\nu)} \int_{0}^{\infty} \frac{u^{\nu-1}}{[1+\beta(1-\alpha) u]_{+}^{\frac{1}{1-\alpha}}} d u
$$

The integral therein involved is

$$
\mathcal{Z}=\frac{\pi^{v}}{[\beta(\alpha-1)]^{v}} \frac{\Gamma\left(\frac{\alpha}{\alpha-1}\right)}{\Gamma\left(\frac{\alpha}{\alpha-1}+v\right)}
$$

Also, for the mean energy $\langle\mathcal{U}\rangle$, we have from $[72$

$$
<\mathcal{U}>=\frac{\pi^{v}}{\Gamma(v) \mathcal{Z}} \int_{0}^{\infty} \frac{u^{v}}{[1+\beta(1-\alpha) u]_{+}^{\frac{1}{1-\alpha}}} d u
$$

whose result is

$$
<\mathcal{U}>=\frac{v \pi^{v}}{\mathcal{Z}[\beta(\alpha-1)]^{v+1}} \frac{\Gamma\left(\frac{\alpha}{\alpha-1}\right)}{\Gamma\left(\frac{\alpha}{\alpha-1}+v+1\right)},
$$

or equivalently,

$$
<\mathcal{U}>=\frac{v}{\beta[\alpha+v(\alpha-1)]}
$$

The entropy can be expressed via $\mathcal{Z}$ and $\langle\mathcal{U}\rangle$ as

$$
\mathcal{S}=\ln \mathcal{Z}+\frac{1}{1-\alpha} \ln [1+(1-\alpha) \beta<\mathcal{U}>
$$

Using (3.2) - (3.4) we cast $\mathcal{S}$ as

$$
\mathcal{S}=\ln \left\{\left[\frac{\pi}{\beta(\alpha-1)}\right]^{\nu} \frac{\Gamma\left(\frac{\alpha}{\alpha-1}\right)}{\Gamma\left(\frac{\alpha}{\alpha-1}+\nu\right)}\right\}+\frac{1}{1-\alpha} \ln \left[\frac{\alpha}{[\alpha+v(\alpha-1)]}\right]
$$

We gather from (2.2) that $\mathcal{Z}$ is positive and finite for $\alpha>1$. 


\section{$2.20<\alpha<1$}

Instead, for $0<\alpha<1$ one has

$$
\mathcal{Z}=\frac{\pi^{v}}{\Gamma(v)} \int_{0}^{\infty} \frac{u^{v-1}}{[1+\beta(1-\alpha) u]^{\frac{1}{1-\alpha}}} d u
$$

and

$$
\mathcal{Z}=\frac{\pi^{v}}{[\beta(1-\alpha)]^{v}} \frac{\Gamma\left(\frac{1}{1-\alpha}-v\right)}{\Gamma\left(\frac{1}{1-\alpha}\right)} .
$$

Let us pass now to the mean energy. For $\langle\mathcal{U}\rangle$ we have

$$
<\mathcal{U}>=\frac{\pi^{v}}{\Gamma(v) \mathcal{Z}} \int_{0}^{\infty} \frac{u^{v}}{[1+\beta(1-\alpha) u]^{\frac{1}{1-\alpha}}} d u
$$

or

$$
<\mathcal{U}>=\frac{v \pi^{\nu}}{\mathcal{Z}[\beta(1-\alpha)]^{v+1}} \frac{\Gamma\left(\frac{1}{1-\alpha}-v-1\right)}{\Gamma\left(\frac{1}{1-\alpha}\right)},
$$

that can be recast as

$$
<\mathcal{U}>=\frac{v}{\beta[\alpha-v(1-\alpha)]}
$$

Here

$$
\mathcal{S}=\ln \left\{\left[\frac{\pi}{\beta(1-\alpha)}\right]^{v} \frac{\Gamma\left(\frac{1}{1-\alpha}-v\right)}{\Gamma\left(\frac{1}{1-\alpha}\right)}\right\}+\frac{1}{1-\alpha} \ln \left[\frac{\alpha}{[\alpha-\nu(1-\alpha)]}\right]
$$

Here we find poles in the partition function.

Outside these poles we have

$$
\frac{1}{1-\alpha}-v<0 \quad ; \quad \Gamma\left(\frac{1}{1-\alpha}-v\right)>0 .
$$

As a consequence, the partition function is both positive and finite. We use the equality

$$
\Gamma\left(\frac{1}{1-\alpha}-v\right)=-\frac{\pi}{\sin \pi\left(v-\frac{1}{1-\alpha}\right) \Gamma\left(v+1-\frac{1}{1-\alpha}\right)}
$$


and ascertain that

$$
\sin \pi\left(v-\frac{1}{1-\alpha}\right)<0
$$

Thus,

$$
2 p+1<v-\frac{1}{1-\alpha}<2 p+2 \quad ; \quad p=0,1,2,3, \ldots \ldots
$$

This chain of inequalities shows that $\alpha$ and $v$ are related to each other.

\section{The divergences of the theory}

Remember beforehand the well known fact that a classical entropy is defined only up to an arbitrary constant. From (2.9), $\mathcal{Z}$ ' poles arise when the Gamma arguments become [73]

$$
\frac{1}{1-\alpha}-v=-p \text { for } p=0,1,2,3, \ldots \ldots,
$$

or, equivalently, for

$$
\alpha=\frac{1}{2}, \frac{2}{3}, \frac{3}{4}, \frac{4}{5}, \ldots \ldots, \frac{v-2}{v-1}, \frac{v-1}{v} .
$$

For $<\mathcal{U}>$ 's poles we have

$$
\frac{1}{1-\alpha}-v-1=-p \text { for } p=0,1,2,3, \ldots \ldots,
$$

or, equivalently,

$$
\alpha=\frac{1}{2}, \frac{2}{3}, \frac{3}{4}, \frac{4}{5}, \ldots \ldots, \frac{v-1}{v}, \frac{v}{v+1} .
$$

\section{The one-dimensional scenario}

In one dimension $\mathcal{Z}$ is regular and $\langle\mathcal{U}\rangle$ has a singularity at $\alpha=\frac{1}{2}$. For $\alpha \neq \frac{1}{2}, \mathcal{Z}$ and $\langle\mathcal{U}\rangle$ can be easily evaluated. The result is straightforward

$$
\mathcal{Z}=\frac{\pi}{\beta \alpha}
$$




$$
<\mathcal{U}>=\frac{1}{\beta(2 \alpha-1)} .
$$

As a consequence, we have for $\mathcal{S}$

$$
\mathcal{S}=\ln \left(\frac{\pi}{\beta \alpha}\right)+\frac{1}{1-\alpha} \ln \left(\frac{\alpha}{2 \alpha-1}\right)
$$

When $\alpha=\frac{1}{2}$, we have for $\mathcal{Z}$

$$
\mathcal{Z}=\frac{2 \pi}{\beta}
$$

a regular value. Regularization is needed then only for $\langle\mathcal{U}\rangle$.

\subsection{Dealing with the divergences}

In order to proceed with such regularizing procedure, the main idea is to write $\langle\mathcal{U}\rangle$ as a function of the dimension $v$ in the fashion

$$
<\mathcal{U}>_{v}=\frac{2^{v+1} v \pi^{v}}{\mathcal{Z} \beta^{v+1}} \Gamma(1-v)
$$

and carefully dissect this expression. We note first that

$$
\Gamma(1-v)=-\frac{1}{v-1}+\mathbf{C}+\sum_{k=1}^{\infty} b_{k}(v-1)^{k}
$$

where $\mathbf{C}$ is Euler's constant. Let

$$
f(v)=\frac{2^{v+1} v \pi^{v}}{\beta^{v+1}}
$$

The Laurent expansion of $f(v)$ in $v=1$ is

$$
f(v)=\frac{4 \pi}{\beta^{2}}+\frac{4 \pi}{\beta^{2}}\left[1+\ln \left(\frac{2 \pi}{\beta}\right)\right](v-1)+\sum_{k=2}^{\infty} c_{k}(v-1)^{k} .
$$

Using (4.6) - (4.8) we obtain

$$
<\mathcal{U}>_{v}=\frac{1}{\mathcal{Z}}\left\{\frac{4 \pi}{\beta^{2}(1-v)}+\frac{4 \pi}{\beta^{2}}\left[\mathbf{C}-1-\ln \left(\frac{2 \pi}{\beta}\right)\right]+\sum_{k=2}^{\infty} d_{k}(v-1)^{k}\right\} .
$$


Use now the $\mathcal{Z}$ value of (4.4) and find

$$
<\mathcal{U}>_{v}=\frac{2}{\beta(1-v)}+\frac{2}{\beta}\left[C-1-\ln \left(\frac{2 \pi}{\beta}\right)\right]+\sum_{k=1}^{\infty} a_{k}(v-1)^{k} .
$$

Dimensional regularization's prescriptions assert that the $\langle\mathcal{U}>$-physical value is given by the $v-1$-independent term in

$$
<\mathcal{U}>=\frac{2}{\beta}\left[\mathrm{C}-1-\ln \left(\frac{2 \pi}{\beta}\right)\right] .
$$

Using then (4.4) - (4.11) we find

$$
\mathcal{S}=\ln \left\{\frac{2 \pi}{\beta}\left[\mathbf{C}-\ln \left(\frac{2 \pi}{\beta}\right)\right]^{2}\right\} .
$$

\section{The two-dimensional case}

For two dimensions, $\mathcal{Z}$ has a singularity at $\alpha=\frac{1}{2}$ and $\langle\mathcal{U}\rangle$ has singularities at $\alpha=\frac{1}{2}$ and $\alpha=\frac{2}{3}$. Save for the case of these singularities, we can evaluate their values of the main statistical quantities without the use of dimensional regularization. Thus, we obtain

$$
\begin{gathered}
\mathcal{Z}=\frac{\pi^{2}}{\beta^{2} \alpha(2 \alpha-1)}, \\
<\mathcal{U}>=\frac{2}{\beta(3 \alpha-2)}, \\
\mathcal{S}=\ln \left[\frac{\pi^{2}}{\beta^{2} \alpha(2 \alpha-1)}\right]+\frac{1}{1-\alpha} \ln \left(\frac{\alpha}{3 \alpha-2}\right)
\end{gathered}
$$

\subsection{The $\alpha=1 / 2$ pole}

For $\alpha=\frac{1}{2}$ we must employ the treatment of the preceding Section, i.e., regularize, both $\mathcal{Z}$ and $\mathcal{U}$. We start with $\mathcal{Z}$

$$
\mathcal{Z}=\left(\frac{2 \pi}{\beta}\right)^{v} \Gamma(2-v)
$$


We recall that

$$
\Gamma(2-v)=-\frac{1}{v-2}+\mathbf{C}+\sum_{k=1}^{\infty} b_{k}(v-2)^{k}
$$

and define

$$
f(v)=\frac{2^{v} \pi^{v}}{\beta^{v}}
$$

The associated Laurent expansion is

$$
f(v)=\frac{4 \pi^{2}}{\beta^{2}}+\frac{4 \pi^{2}}{\beta^{2}} \ln \left(\frac{2 \pi}{\beta}\right)(v-2)+\sum_{k=2}^{\infty} c_{k}(v-2)^{k} .
$$

Using (5.5) - (5.7) we find

$$
\mathcal{Z}_{v}=-\frac{4 \pi^{2}}{\beta^{2}(v-2)}+\frac{4 \pi^{2}}{\beta^{2}}\left[C-\ln \left(\frac{2 \pi}{\beta}\right)\right]+\sum_{k=1}^{\infty} a_{k}(v-2)^{k},
$$

and the physical value for he partition function becomes

$$
\mathcal{Z}=\frac{4 \pi^{2}}{\beta^{2}}\left[\mathbf{C}-\ln \left(\frac{2 \pi}{\beta}\right)\right] .
$$

Since $\mathcal{Z}$ must be positive, we find the following upper bound for $\mathrm{T}$

$$
\mathrm{T}<\frac{e^{\mathrm{c}}}{2 \pi \mathrm{k}}
$$

For $\mathcal{U}$ the situation is similar. From (3.4) we have

$$
<\mathcal{U}>=\frac{v}{\mathcal{Z} \pi}\left(\frac{2 \pi}{\beta}\right)^{v+1} \Gamma(1-v) .
$$

Define

$$
f(v)=\frac{v 2^{v+1} \pi^{v}}{\beta^{v+1}(1-v)}
$$

The pertinent Laurent expansion is

$$
f(v)=-\frac{16 \pi^{2}}{\beta^{2}}+\frac{16 \pi^{2}}{\beta^{2}}\left[\frac{1}{2}-\ln \left(\frac{2 \pi}{\beta}\right)\right](v-2)+\sum_{k=2}^{\infty} c_{k}(v-2)^{k} .
$$


From (5.5) - (5.13) we find

$$
<\mathcal{U}>_{v}=\frac{1}{\mathcal{Z}}\left\{\frac{16 \pi^{2}}{\beta^{3}(v-2)}+\frac{16 \pi^{2}}{\beta^{3}}\left[\ln \left(\frac{2 \pi}{\beta}\right)-C-\frac{1}{2}\right]+\sum_{k=1}^{\infty} a_{k}(v-2)^{k}\right\} .
$$

Thus, $\mathcal{U}$ 's physical value is (remember 5.10)

$$
<\mathcal{U}>=\frac{4}{\beta} \frac{\ln \left(\frac{2 \pi}{\beta}\right)-\mathbf{C}-\frac{1}{2}}{\mathrm{C}-\ln \left(\frac{2 \pi}{\beta}\right)} .
$$

Using (5.9) - (5.15) we find

$$
\mathcal{S}=\ln \left\{\frac{4 \pi^{2}}{\beta^{2}} \frac{\left[\ln \left(\frac{2 \pi}{\beta}\right)-\mathbf{C}-1\right]^{2}}{\mathrm{C}-\ln \left(\frac{2 \pi}{\beta}\right)}\right\} .
$$

\subsection{The $\alpha=2 / 3$ pole}

For $\alpha=\frac{2}{3}, \mathcal{Z}$ is finite and $\langle\mathcal{U}\rangle$ has a pole. The procedure for finding their physical values is similar to that for the case $\alpha=\frac{1}{2}$. For this reason, we merely indicate the results obtained for $\mathcal{Z},\langle\mathcal{U}\rangle$, and $\mathcal{S}$. One finds

$$
\begin{gathered}
\mathcal{Z}=\frac{9 \pi^{2}}{2 \beta^{2}}, \\
<\mathcal{U}>=\frac{6}{\beta}\left[\mathrm{C}-\frac{1}{2}-\ln \left(\frac{3 \pi}{\beta}\right)\right], \\
\mathcal{S}=\ln \left\{\frac{36 \pi^{2}}{\beta^{2}}\left[\mathrm{C}-\ln \left(\frac{2 \pi}{\beta}\right)\right]^{3}\right\}
\end{gathered}
$$

\section{The three-dimensional instance}

In three dimensions, $\mathcal{Z}$ has poles at $\alpha=\frac{1}{2}$ and $\alpha=\frac{2}{3}$ while $\langle\mathcal{U}\rangle$ exhibits them at $\alpha=\frac{1}{2}, \alpha=\frac{2}{3}$, and $\alpha=\frac{3}{4}$. Outside the poles one has for $\mathcal{Z},\langle\mathcal{U}\rangle$, and $\mathcal{S}$, respectively,

$$
\mathcal{Z}=\frac{\pi^{3}}{\beta^{3} \alpha(2 \alpha-1)(3 \alpha-2)}
$$




$$
\begin{gathered}
<\mathcal{U}>=\frac{3}{\beta(4 \alpha-3)} . \\
\mathcal{S}=\ln \left[\frac{\pi^{3}}{\beta^{3} \alpha(2 \alpha-1)(3 \alpha-2)}\right]+\frac{1}{1-\alpha} \ln \left(\frac{\alpha}{4 \alpha-3}\right)
\end{gathered}
$$

In this case $\alpha$ should satisfy the condition $\alpha<\frac{5}{4}$ for the mean energy to be a positive quantity.

\subsection{The $\alpha=1 / 2$ pole}

For $\alpha=\frac{1}{2}$ we have

$$
\mathcal{Z}_{v}=\left(\frac{2 \pi}{\beta}\right)^{v} \Gamma(2-v) .
$$

The Laurent expansion is tackled as above. One finds

$$
\mathcal{Z}_{v}=-\frac{8 \pi^{3}}{\beta^{3}(v-3)}+\frac{8 \pi^{3}}{\beta^{3}}\left[\ln \left(\frac{2 \pi}{\beta}-1-C\right)\right]+\sum_{k=1}^{\infty} a_{k}(v-3)^{k}
$$

From (6.5) it is easy to obtain the physical value of $\mathcal{Z}$ as

$$
\mathcal{Z}=\frac{8 \pi^{3}}{\beta^{3}}\left[\ln \left(\frac{2 \pi}{\beta}\right)-1-\mathrm{C}\right]
$$

Since $\mathcal{Z}$ is positive, one is led to the bound

$$
\mathrm{T}>\frac{e^{\mathrm{C}+1}}{2 \pi \mathrm{k}}
$$

In a similar vein, we have for $\langle\mathcal{U}\rangle$

$$
<\mathcal{U}>=\frac{1}{\beta} \frac{\frac{7}{2}+3 \mathrm{C}-3 \ln \left(\frac{2 \pi}{\beta}\right)}{\ln \left(\frac{2 \pi}{\beta}\right)-\mathrm{C}-1}
$$

and from (6.6) and (6.8)

$$
\mathcal{S}=\ln \left\{\frac{2 \pi^{3}}{\beta^{3}} \frac{\left[2 \mathrm{C}+3-2 \ln \left(\frac{2 \pi}{\beta}\right)\right]^{2}}{\ln \left(\frac{2 \pi}{\beta}\right)-1-\mathrm{C}}\right\}
$$




\subsection{The $\alpha=2 / 3$ and $\alpha=3 / 4$ poles}

For $\alpha=\frac{2}{3}$ and $\alpha=\frac{3}{4}$ we give only the corresponding results, since the calculations are entirely similar to those for the case $\alpha=\frac{1}{2}$. Thus, for ,$\alpha=\frac{2}{3}$ we have

$$
\mathcal{Z}=\frac{27 \pi^{3}}{2 \beta^{3}}\left[\mathbf{C}-\ln \left(\frac{3 \pi}{\beta}\right)\right]
$$

Here one requires

$$
\begin{gathered}
\mathrm{T}<\frac{e^{\mathrm{C}}}{3 \pi \mathrm{k}} \\
<\mathcal{U}>=\frac{1}{\beta} \frac{9 \ln \left(\frac{3 \pi}{\beta}\right)-6-9 \mathrm{C}}{\mathrm{C}-\ln \left(\frac{3 \pi}{\beta}\right)} \\
\mathcal{S}=\ln \left\{\frac{27 \pi^{3}}{2 \beta^{3}} \frac{\left[\ln \left(\frac{9 \pi^{2}}{\beta^{2}}\right)-2-2 \mathrm{C}\right]^{3}}{\left[\mathrm{c}-\ln \left(\frac{2 \pi}{\beta}\right)\right]^{2}}\right\}
\end{gathered}
$$

For $\alpha=\frac{3}{4}$ we have

$$
\begin{gathered}
\mathcal{Z}=\frac{32 \pi^{3}}{3 \beta^{3}} \\
<\mathcal{U}>=\frac{4}{\beta}\left[3 \boldsymbol{C}-1-3 \ln \left(\frac{4 \pi}{\beta}\right)\right], \\
\mathcal{S}=\ln \left\{\frac{5 \pi^{3}}{\beta^{3}}\left[\mathbf{C}-\ln \left(\frac{4 \pi}{\beta}\right)\right]^{4}\right\} .
\end{gathered}
$$

\section{Specific Heats}

We set $k \equiv k_{\mathrm{B}}$. For $v=1$, in the regular case we have for the specific heat C:

$$
\mathcal{C}=\frac{k}{2 \alpha-1}
$$

For $v=2$ one has

$$
\mathcal{C}=\frac{2 k}{3 \alpha-2}
$$


Finally, for $v=3$ one ascertains that

$$
\mathcal{C}=\frac{3 k}{4 \alpha-3},
$$

\subsection{Specific heats at the poles}

For $\vee=1 ; \alpha=\frac{1}{2}$

$$
\mathcal{C}=2 k(\mathbf{C}-2-\ln 2 \pi k T) .
$$

For $v=2 ; \alpha=\frac{1}{2}$

$$
\mathcal{C}=\frac{2 k(2 \ln 2 \pi k T-1-2 \mathbf{C})}{\mathbf{C}-\ln 2 \pi k T}-\frac{2 k}{(\mathbf{C}-\ln 2 \pi k T)^{2}} .
$$

For $v=2$ and $\alpha=\frac{2}{3}$ one has

$$
\mathcal{C}=6 k\left(\mathrm{C}-\frac{3}{2}-\ln 3 \pi k T\right)
$$

For $v=3 ; \alpha=\frac{1}{2}$,

$$
\mathcal{C}=k \frac{3 \mathbf{C}+\frac{7}{2}-3 \ln 2 \pi k T}{\ln 2 \pi k T-\mathbf{C}-1}-\frac{k}{2(\ln 2 \pi k T-\mathbf{C}-1)^{2}} .
$$

For $v=3$ and $\alpha=\frac{2}{3}$ one finds

$$
\mathcal{C}=k \frac{9 \ln 3 \pi k T-6-9 \mathbf{C}}{\mathbf{C}-\ln 3 \pi k T}-\frac{6 k}{(\mathbf{C}-\ln 3 \pi k T)^{2}} .
$$

Finally, for $v=3$ and $\alpha=\frac{3}{4}$ we obtain

$$
\mathcal{C}=4 k(3 \mathrm{C}-4-3 \ln 4 \pi \mathrm{kT}) .
$$

Figs, 1, 2, and 3 plot the mean energy's pole-specific heats, within their allowed temperature ranges, for one, two, and three dimensions, respectively. The most distinguished feature emerges in the cases in which we deal with $<$ $\mathrm{U}>-$ poles for which $\mathrm{Z}$ is regular. We see in such a case that negative specific heats arise. Such an occurrence has been associated to self-gravitational systems [74]. In turn, Verlinde has associated this type of systems to an 
entropic force [75]. It is natural to conjecture then that such a force may appear at the energy associated poles.

Notice also that temperature ranges are restricted. There is an $\mathrm{T}$-upper bound, and one may wish to remember, in this respect, the notion of Hagedorn temperature [76], as an example a temperature's upper bound. In two and three dimensions there is also a lower bound, so that the system (at the poles) would be stable only in a limited $\mathrm{T}$-range.

\section{Discussion}

In this work we have appealed to an elementary regularization procedure to study the poles in the partition function and the mean energy that appear, for specific, discrete q-values, in Renyi's statistics of the harmonic oscillator. We studied the thermodynamic behavior at the poles and found interesting peculiarities. The analysis was made in one, two, three, and 3 dimensions. Amongst the pole-traits we emphasize:

- The poles appear, both in the partition function and the mean energy, for $0<\alpha<1$.

- These poles ar an artifact of having $\alpha \neq 1$.

- We have proved that there is an upper bound to the temperature at the poles, confirming the findings of Ref. [77], in the sense that, for $\alpha \neq 1$, the heath bath of the canonical ensemble must be finite.

- In some cases, Renyi's' entropies are positive only for a restricted temperature-range. Lower $\mathrm{T}$ bounds seem to be a new trait discovered here.

- Negative specific heats, characteristic trait of self-gravitating systems, are encountered.

Our physical results derive only from statistics, not from mechanical effects. This fact reminds us of a similar occurrence in the case of the entropic force conjectured by Verlinde [75].

Indeed, the poles arise only because $\alpha \neq 1$. They are a property of the entropic quantifier, not of the Hamiltonian. Indeed, only for $\alpha \neq 1$ a Gamma 
function appears in the partition function. This Gamma function may display poles.

Future research should be concerned with cases where it is already known in advance that $\alpha \neq 1$. For these cases, the traits here discovered may acquire some degree of physical "reality".

The importance of the present communication resides in that fact of having disclosed Renyi's entropy traits that could not have been suspected before. 


\section{References}

[1] C. Beck and F. Schlogl, Thermodynamics of chaotic systems: an introduction (Cambridge University Press, Cambridge, England, 1993).

[2] C. M. Herdman, Stephen Inglis, P.-N. Roy, R. G. Melko, and A. Del Maestro, Phys. Rev. E 90, 013308 (2014).

[3] Mohammad H. Ansari and Yuli V. Nazarov, Phys. Rev. B 91, 174307 (2015).

[4] Lei Wang and Matthias Troyer, Phys. Rev. Lett. 113, 110401 (2014).

[5] Matthew B. Hastings, Ivn Gonzlez, Ann B. Kallin, and Roger G. Melko, Phys. Rev. Lett 104, 157201 (2010).

[6] Richard Berkovits, Phys. Rev. Lett. 115, 206401 (2015).

[7] Nima Lashkari, Phys. Rev. Lett. 113, 051602 (2014).

[8] Gabor B. Halasz and Alioscia Hamma, Phys. Rev. Lett. 110, 170605 (2013).

[9] MB Hastings, I Gonzlez, AB Kallin and RG Melko, Phys. Rev. Lett. 104, 157201 (2010); A. De Gregorio, S.M. lacus, 179, 279 (2009).

[10] Leila Golshani, Einollah Pasha and Gholamhossein Yari, Information Sciences, 179, 2426 (2009); J.F. Bercher, Information Sciences 178, 2489 (2008).

[11] EK Lenzi, RS Mendes and LR da Silva, Physica A 280, 337 (2000).

[12] A.Plastino, M. C. Rocca, F. Pennini, Phys. Rev. E 94, 012145 (2016).

[13] C. G. Bollini and J. J. Giambiagi, Phys. Lett. B 40, 566 (1972); Il Nuovo Cim. B 12, 20 (1972); W. Bietenholz, L. Prado, Physics Today 67, 38 (2014).

[14] C. G. Bollini and J.J Giambiagi, Phys. Rev. D 53, 5761 (1996).

[15] C. G. Bollini, T. Escobar and M. C. Rocca : Int. J. of Theor. Phys. 38, 2315 (1999). 
[16] C. G Bollini and M.C. Rocca: Int. J. of Theor. Phys. 43, 59 (2004); Int. J. of Theor. Phys. 43, 1019 (2004); Int. J. of Theor. Phys. 46, 3030 (2007).

[17] A. Plastino, M. C. Rocca: "Quantum Field Theory, Feynman and Wheeler Propagators, Dimensional Regularization in Configuration Space, and Convolution of Lorentz Invariant Tempered Distributions". arXiv:1708.04506.

[18] D. Berenstein and A. Miller: Phys. Rev. D 90, 086011 (2014).

[19] D. Anselmi: Phys. Rev. D 89, 125024 (2014).

[20] P. Jaranowski and G. Schfer: Phys. Rev. D 87, 081503(R) (2013).

[21] T. Inagaki, D. Kimura, H. Kohyama, and A. Kvinikhidze: Phys. Rev. D 86, 116013 (2012).

[22] J. Qiu: Phys. Rev. D 77, 125032 (2008).

[23] L. Blanchet, T. Damour, G. Esposito-Farse, and B. R. Iyer: Phys. Rev. D 71, 124004 (2005).

[24] F. Bastianelli, O. Corradini, and A. Zirotti: Phys. Rev. D 67, 104009 (2003).

[25] D. Lehmann and G. Przeau: Phys. Rev. D 65, 016001 (2001).

[26] A. P. Bata Scarpelli, M. Sampaio, and M. C. Nemes: Phys. Rev. D 63, 046004 (2001).

[27] E. Braaten and Yu-Qi Chen: Phys. Rev. D 55, 7152 (1997).

[28] J. Smith and W. L. van Neerven EPJ C 40, 199 (2005).

[29] J. F. Schonfeld EPJ C 76, 710 (2016).

[30] C. Gnendiger et al.: EPJ C 77, 471 (2017).

[31] P. Arnold, Han-Chih Chang and S. Iqbal: JHEP 100 (2016).

[32] I. AravE, Y. Oz and A. Raviv-Moshe: JHEP 88 (2017). 
[33] C. Anastasiou, S. Buehler, C. Duhr and F. Herzog: JHEP 62 (2012).

[34] F. Niedermayer and P. Weisz: JHEP 110 (2016).

[35] C. Coriano, L. Delle Rose, E. Mottolaand M. Serino: JHEP 147 (2012).

[36] F. Dulat, S. Lionetti, B. Mistlberger,A. Pelloni and C. Specchia: JHEP 17 (2017).

[37] T. Gehrmann and N. Greiner: JHEP 50 (2010).

[38] T.Lappia and R.Paatelainena: Ann. of Phys. 379, 34 (2017).

[39] S.Grooteab, J.G.Krner and A.A.Pivovarov: Ann. of Phys. 322, 2374 (2007).

[40] N.C.Tsamis and R.P.Woodard: Ann. of Phys. 321, 875 (2006).

[41] S. Krewaland and K. Nakayama: Ann. of Phys. 216, 210 (1992).

[42] L. Rosen and J. D. Wright Comm. Math. Phys. 134, 433 (1990).

[43] F. David Comm. Math. Phys. 81, 149 (1981).

[44] P. Breitenlohner and D. Maison Comm. Math. Phys. 52, 11 (1977).

[45] S. Teber and A. V. Kotikov: EPL 107, 57001 (2014).

[46] H. Fujisaki: EPL 28, 623 (1994).

[47] M. W. Kalinowski, M. Seweryski and L. Szymanowski: JMP 24, 375 (1983).

[48] R. Contino and A. Gambassi: JMP 44, 570 (2003).

[49] M. Dutsch, K. Fredenhagen, K. J. Keller and K. Rejzner3: JMP 55, 122303 (2014).

[50] T. Nguyena: JMP 57, 092301 (2016).

[51] J. Ben Geloun and R. Toriumi: JMP 56, 093503 (2015).

[52] J. Ben Geloun and R. Toriumi: J. Phys. A 45, 374026 (2012). 
[53] B. Mutet, P. Grange and E. Werner: J. Phys. A 45, 315401 (2012).

[54] M. C Abbott and P. Sundin: J. Phys. A 45, 025401 (2012).

[55] T Fujihara et al.: J. Phys. A 39, 6371 (2008).

[56] Silke Falk et al.: J. Phys. A 43, 035401 (2010).

[57] Germn Rodrigo et al.: J. Phys. G 25, 1593 (1999).

[58] B. M. Pimentel and J. L. Tomazelli: J. Phys. G 20, 845 (1994).

[59] A. Khare: J. Phys. G 3, 1019 (1977).

[60] J. C. D'Cruz: J. Phys. G 1, 151 (1975).

[61] R. Sepahv and S. Dadfar: Nuc. Phys. A 960, 36 (2017).

[62] J. V. Steele and R. J. Furnstahl: Nuc. Phys. A 630, 46 (1998).

[63] D. R. Phillips, S. R. Beane and T. D. Cohena: Nuc. Phys. A 631, 447 (1998).

[64] A. J. Stoddart and R. D. Viollier: Nuc. Phys. A 532, 657 (1991).

[65] E. Panzer: Nuc. Phys. B 874, 567 (2013).

[66] R. N. Lee, A. V. Smirnov and and V. A. Smirnov: Nuc. Phys. B 856, 95 (2012).

[67] A. P. Isaev: Nuc. Phys. B 662, 461 (2003).

[68] J. M. Campbell, E. W. N. Glover and D. J. Miller: Nuc. Phys. B 498, 397 (1997).

[69] C. J. Yang, M. Grasso, X. Roca-Maza, G. Colo, and K. Moghrabi: Phys. Rev. C 94, 034311 (2016).

[70] K. Moghrabi and M. Grasso: Phys. Rev. C 86, 044319 (2012).

[71] D. R. Phillips, I. R. Afnan, and A. G. Henry-Edwards: Phys. Rev. C 61, $044002(2000)$.

[72] A. Plastino and M. C. Rocca Europhysics Letters 104, 60003 (2013). 
[73] These poles emerge because a Gamma function enters the partition function. As an example see: A. Plastino and M. C. Rocca: arXiv:1702.03535

[74] D. Lynden-Bell and R. M. Lynden-Bell, Mon. Not. R. Astron. Soc. 181, 405 (1977).

[75] E. Verlinde, arXiv:1001.0785 [hep-th]; JHEP 04, 29 (2011).

[76] J. J. Atick and E. Witten, Nucl. Phys. B. 310, 291 (1988).

[77] A.R. Plastino and A. Plastino, Phys. Lett. A 193, 140 (1994). 


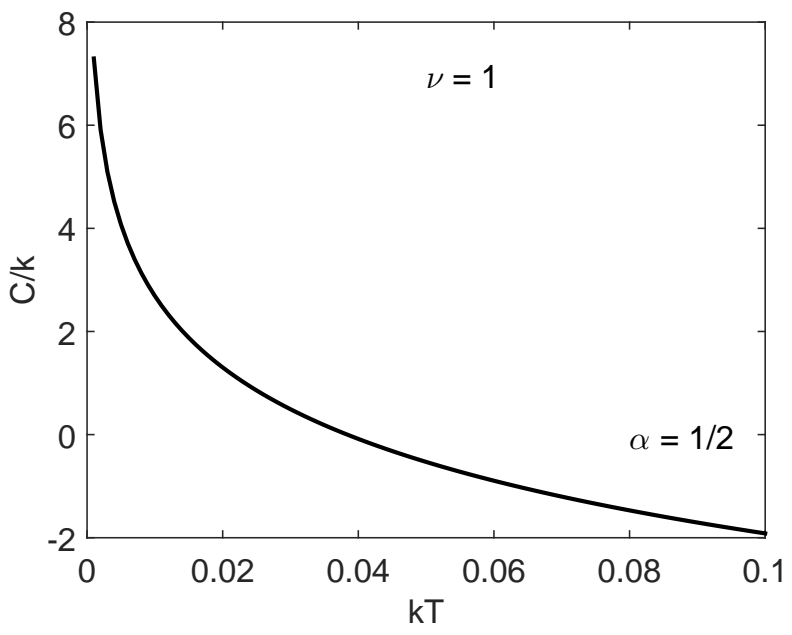

Figure 1: One dimension: specific heats at the pole versus temperature $\mathrm{T}$. 


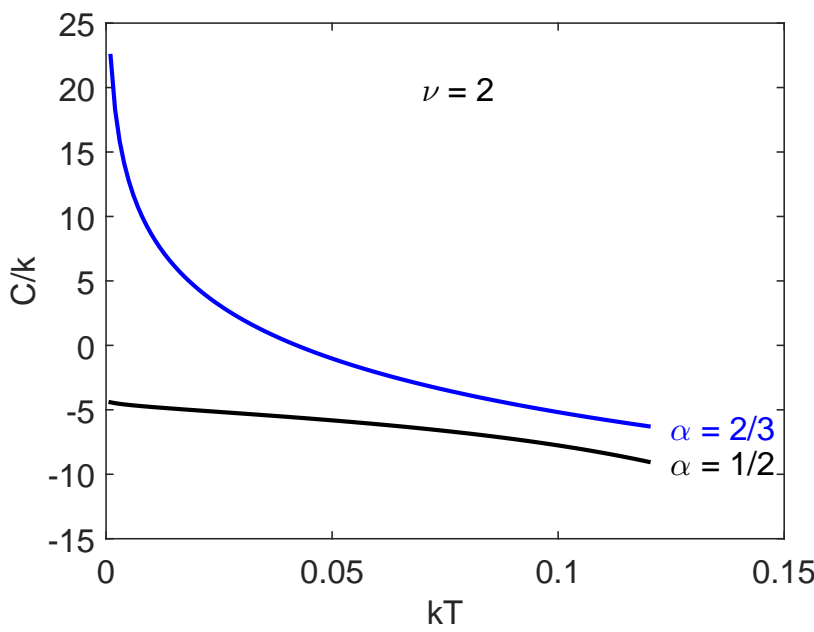

Figure 2: Two dimensions: specific heats at the two poles versus temperature T. 


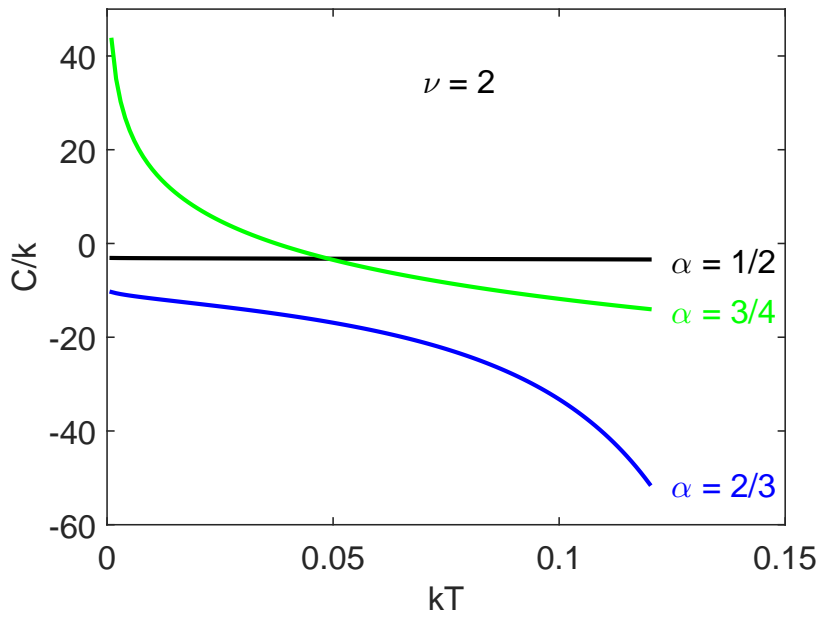

Figure 3: Three dimensions: specific heats at the three poles versus temperature $\mathrm{T}$. 